\title{
Characterization of a novel ADP-ribosylation factor gene from Macrobrachium nipponense and its response to ammonia nitrogen stress
}

\author{
Leifeng Guo ${ }^{1, \#}$, Bing Sun ${ }^{2, \#}$, Duanduan Chen ${ }^{1}$, Cao Yi ${ }^{1}$, Jian Teng ${ }^{3}$, Jielun Yu ${ }^{4}$, \\ Shouquan Wang ${ }^{1}$, Yuanyuan $\mathrm{Ru}^{1}$, Hui Wang ${ }^{1, *}$ \\ ${ }^{1}$ Department of Aquaculture Research Lab, College of Animal Science and Veterinary Medicine, \\ Shandong Agricultural University, Taian 271018, PR China \\ ${ }^{2}$ College of Fisheries, Key Lab of Agricultural Animal Genetics, Breeding, and Reproduction of Ministry of Education, \\ Huazhong Agricultural University, No. 1 Shizishan Street, Hongshan District, Wuhan 430070, Hubei, PR China \\ ${ }^{3}$ College of Marine Sciences, Ningbo University, Ningbo, Zhejiang 315211, PR China \\ ${ }^{4}$ Experimental Center for Medical Research, Weifang Medical University, Weifang 261053, PR China
}

\begin{abstract}
ADP-ribosylation factors (Arfs) are guanosine triphosphate (GTP)-binding proteins that play essential roles in membrane trafficking, and they have been recently reported to be involved in innate immunity in crustaceans. However, little information is available on Arfs in the oriental river prawn Macrobrachium nipponense and their response to ammonia nitrogen stress. In this study, we identified a novel M. nipponense Arfn gene (MnArfn). The full-length cDNA of MnArfn was $1076 \mathrm{bp}$. It contained a $537 \mathrm{bp}$ open reading frame (ORF) and encoded a 178 amino acid protein with a predicted molecular weight of $19.85 \mathrm{kDa}$. Sequence and phylogenetic analyses showed that MnArfn was an unidentified Arf, sharing 55-61\% identity with other known Arfs. Quantitative real-time PCR (qPCR) indicated that all examined tissues (hepatopancreas, stomach, gill, heart, muscle, and eyestalk) expressed MnArfn. Hepatopancreas and gills, 2 organs involved in environmental stress management, had the highest expression. Under conditions of ammonia nitrogen stress, MnArfn expression in hepatopancreas and gills was significantly up-regulated at 6,12 , and $24 \mathrm{~h}$. Western blotting experiments also revealed that MnArfn was distributed in all examined tissues, with the highest expression in hepatopancreas and gills, consistent with qPCR results. The findings from this study indicate that MnArfn may play an important role in the response of $M$. nipponense to ammonia nitrogen stress, which provides a new avenue to study the resistance mechanism(s) of crustaceans to ammonia nitrogen and to screen for individuals with resistance to unfavorable environments.
\end{abstract}

KEY WORDS: Macrobrachium nipponense $\cdot$ ADP ribosylation factor $\cdot$ Ammonia nitrogen stress

\section{INTRODUCTION}

The oriental river prawn Macrobrachium nipponense (Palaemonidae) (Cui et al. 2018) is an economically important aquacultural crustacean bred in freshwater and low-salinity estuarine regions in China (Cheng et al. 2017). However, with the ex-

*Corresponding author: wanghui2328@sdau.edu.cn

\#These authors contributed equally to this work pansion of culture practices and the deterioration of ecological environments (Duan et al. 2013, Price et al. 2015), environmental stresses have become the main restricting factor for the development of the prawn industry, and in some cases, large-scale economic losses have been reported (Moraes-Valenti \& Valenti 2007, Lu et al. 2016). Ammonia nitrogen is Attribution restricted. Authors and original publication must be credited. 
the main toxic component in aquaculture water (Chen et al. 2016, Liang et al. 2016), and previous studies have reported that an elevated ammonia nitrogen level can impair the immune system (Chen \& Kou 1992, Cheng \& Chen 2002, Yue et al. 2010) and affect oxygen consumption (Chen \& Lin 1992) in crustaceans. Moreover, the accumulation of excreted ammonia nitrogen can induce the expression of stress response-related and immunerelated genes such as heat shock protein 90 (HSP90) (Li et al. 2012), ADP-ribosylation factor 1 (Arf1) (Duan et al. 2016), and a multiligand-binding protein, $g C 1 q R$ (Sun et al. 2019). Therefore, a better understanding of the response of prawns to ammonia nitrogen stress is critical.

ADP-ribosylation factors (Arfs) are guanosine triphosphate (GTP)-binding proteins that play essential roles in membrane trafficking and cytoskeleton remodeling (Myers \& Casanova 2008, Ding et al. 2015). Arfs are present in organisms including yeast, bacteria, vertebrates, and invertebrates (Ding et al. 2015). Based on sequence analyses, the 6 mammalian Arfs can be classified into 3 categories: class I comprises Arfs 1-3, class II comprises Arfs 4 and 5, and class III includes only Arf 6 (Zhang et al. 2010). Furthermore, different Arf proteins have different intracellular localizations and functions, depending on their membrane components and the diverse group of proteins they recruit (Donaldson \& Honda 2005, D'SouzaSchorey \& Chavrier 2006). Arfs exist in 2 conformations, namely GTP- and guanine diphosphate (GDP)-bound forms, and they are catalyzed by guanine exchange factors (GEFs) and GTPase activating proteins (GAPs). Recently, it has been reported that members of the small GTPase protein family, such as Rho, Ran (Liu et al. 2009), and Rab (Wu et al. 2008), are involved in the defense response in shrimp (He et al. 2004, Pan et al. 2005). Furthermore, shrimp Arfs have been found to participate in virus invasion and/or host defense (Wang et al. 2009).

Although several Arfs have been characterized from various organisms, the function of $M$. nipponense MnArfn in ammonia nitrogen stress is unclear. In this study, a novel Arf gene (MnArfn) from M. nipponense was identified and compared with known Afrs for the first time. The expression of MnAfrn gene in different tissues was also analyzed. We hope that our results will reveal the role of MnAfrn in ammonia nitrogen stress and provide a theoretical basis for future studies on the mechanism(s) of the MnAfrn gene.

\section{MATERIALS AND METHODS}

\subsection{Ethics statement}

This research was conducted in strict accordance with the International Guiding Principles for Biomedical Research Involving Animals 2012 (Council for International Organizations of Medical Sciences, www.cioms.ch), which specifies the care and permissible uses of experimental animals. The research did not involve endangered or protected species.

\subsection{Animals}

Healthy adult Macrobrachium nipponense, with an average weight of $3.71 \pm 0.5 \mathrm{~g}$ (mean $\pm \mathrm{SD}$ ), were obtained from the Huangqian Reservoir in Taian, Shandong Province, China. Prawns were maintained in $800 \mathrm{l}$ plastic tanks with aerated freshwater (temperature: $26 \pm 0.5^{\circ} \mathrm{C}, \mathrm{pH}: 7.8-8.2$ ) and fed daily with a ration corresponding to $4 \%$ of the body weight for $1 \mathrm{wk}$ before the experiments.

\subsection{Ammonia nitrogen challenge and sampling}

The ammonia nitrogen group and the control group each included 150 randomly selected prawns. According to the results of a $48 \mathrm{~h}$ median lethal concentration test, based on a pre-experiment containing 150 samples, an ammonia nitrogen dose of $98 \mathrm{mg}$ $\mathrm{l}^{-1}$ was used in this study. Nessler's reagent was used to measure the total ammonia nitrogen and $\mathrm{NH}_{4} \mathrm{Cl}$ levels, and then both components were adjusted to their desired concentrations. 150 healthy prawns were cultured under conditions of ammonia nitrogen stress for $96 \mathrm{~h}$. Six individuals were selected from the treatment and control groups at 8 time points of 0,3 , $6,12,24,48,72$, and $96 \mathrm{~h}$ (a total of 96 individuals were collected in this study) for hepatopancreas, gill, stomach, heart, muscle, eyestalk, and all samples were stored at $-80^{\circ} \mathrm{C}$ for subsequent testing and analysis of each sample individually.

\subsection{RNA preparation and cDNA synthesis}

Total RNA from tissues collected at all time points was extracted according the manufacturer's instructions for TransZol UP (ET111-01, Trans Gen Biotech). RNA samples were evaluated by $1 \%$ agarose gel electrophoresis, and concentrations were determined 
using a NanoPhotometer spectrophotometer (IMPLEN). First-strand cDNA was synthesized using a PrimeScript ${ }^{\mathrm{TM}}$ RT Reagent Kit (Takara) and the oligo $\mathrm{dT}$ primer from the PrimeScript ${ }^{\mathrm{TM}}$ kit. All cDNAs were stored at $-20^{\circ} \mathrm{C}$ and used as templates in PCR.

\subsection{Cloning the full-length MnArfn cDNA}

A fragment of the MnArfn sequence was obtained from a hepatopancreas cDNA library available in our laboratory that was prepared as described by $\mathrm{Yu}$ et al. (2019). To obtain the full-length MnArfn, genespecific primers (Arfn-5F1, Arfn-5F2, Arfn-3R1, Arfn-3R2) (Table 1) were designed for 5'- and 3'RACE, and the 5' and 3' ends were obtained using a 3'-Full RACE Core Set ver. 2.0 kit and a 5'-Full RACE Kit (Takara), respectively. PCR conditions followed the manufacturer's instructions. PCR products were purified using a gel extraction kit (Sangon Biotech), cloned into the pMD18-T vector (Takara), and sequenced by Sangon Biotech.

\subsection{Sequence analysis}

BLAST (www.ncbi.nlm.nih.gov/BLAST/) was used to analyze and compare nucleotide and deduced amino acid sequences of MnArfn. The open reading frame (ORF) Finder program (www.ncbi.nlm.nih.gov/ projects/gorf/orfig.cgi) was used to deduce the amino acid sequence. The molecular weight and isoelectric point of MnArfn were determined with the 'compute
pI/Mw' tool (http://web.expasy.org/compute_pi/). Multiple sequence alignment was accomplished using the ClustalW Multiple Sequence Alignment program (www.ebi.ac.uk/clustalw/). A phylogenetic tree was constructed using MEGA 7.0 software, and the neighbor-joining method was used for phylogenetic analysis (www.megasoftware.net/mega7/).

\subsection{Expression analysis with quantitative real-time PCR (qPCR)}

qPCR was used to analyze MnArfn expression in the muscle, heart, hepatopancreas, gills, eyestalk, and stomach in a LightCycler ${ }^{\circledR 96}$ system (Roche Diagnostics). Primers (Arfn-F and Arfn-R) (Table 1) were designed based on the nucleotide sequence of MnArfn. Macrobrachium rosenbergii $\beta$-actin (GenBank KY038927.1) was used as an internal control. qPCR was carried out using the TB Green ${ }^{\mathrm{TM}}$ Advantage ${ }^{\circledR} \mathrm{qPCR}$ Premix (Takara) according to the manufacturer's instructions. The qPCR cycling conditions were $95^{\circ} \mathrm{C}$ for $30 \mathrm{~s}$, followed by 40 cycles at $95^{\circ} \mathrm{C}$ for $10 \mathrm{~s}$ and $56^{\circ} \mathrm{C}$ for $30 \mathrm{~s}$. Each sample was assayed 6 times. The $2^{-\Delta \Delta \mathrm{ct}}$ method was used to calculate the expression level of MnArfn.

\subsection{Statistical analysis}

Statistical analysis was performed using SPSS Ver 21.0 (IBM) and GraphPad Prism 8.0 (GraphPad Software) software. We used 1-way ANOVA and

Table 1. Primers used in this study. EcoR-I and Xho-I restriction enzyme sites for Arfn-pro-F and Arfn-pro-R, respectively, are underlined

\begin{tabular}{|c|c|c|}
\hline Primers & Sequence $\left(5^{\prime}-3^{\prime}\right)$ & Purpose \\
\hline $\begin{array}{l}\text { Arfn-5F1 } \\
\text { (outer primer) }\end{array}$ & TCC GTC CAC TTC TAC CAC & 5' RACE \\
\hline $\begin{array}{l}\text { Arfn-5F2 } \\
\text { (inner primer) }\end{array}$ & CAC GGG CGA ACG ATA AAC & \\
\hline $\begin{array}{l}\text { Arfn-3R1 } \\
\text { (outer primer) }\end{array}$ & ACG CCT GAA ACG CCC TTG GT & 3' RACE \\
\hline $\begin{array}{l}\text { Arfn-3R2 } \\
\text { (inner primer) }\end{array}$ & AAG GAT TGG TCT TGA & \\
\hline Arfn-F & CAT CCT GAG CAG CAT TCT & qPCR \\
\hline Arfn-R & CCT TGT CTG TGC TGT CTA CG & \\
\hline$\beta$-actinF $F^{\mathrm{a}}$ & TAT GCA CTT CCT CAT GCC AT & \\
\hline$\beta$-actinR & AGG AGG CGG CAG TGG TCA T & \\
\hline Arfn-pro-F & TAT CGG ATC C GAA TTC ATG GGT GCC ATC CTG AGC & protein analysis \\
\hline Arfn-pro-R & GGT GGT GGT G CTC GA GTC ACT CTG TGA CCG CCT TGG & \\
\hline
\end{tabular}


Tukey's multiple range tests to compare the expression levels of MnArfn in the hepatopancreas and gills to evaluate the differences between the control and treatment groups. All data used for plotting met the ANOVA assumptions (normality and homogeneity of variances).

\subsection{Recombinant MnArfn protein expression and antiserum preparation}

Based on the full-length MnArfn cDNA, primers (Arfn-pro-F and Arfn-pro-R) were designed to amplify the mature peptide. EcoR-I and Xho-I restriction enzyme sites, underlined in Table 1, were selected for Arfn-pro-F and Arfn-pro-R, respectively. The fragment was cloned into the pMD18-T vector (Takara) and sequenced. The fragment was then digested and inserted into the pET-30a (+) vector. The recombinant plasmid (pET30a (+)-MnArfn) was transformed into E. coli BL21 (DE3) cells (Trans Gen Biotech). After induction of expression with IPTG at $25^{\circ} \mathrm{C}$, bacterial cells were harvested, re-suspended in phosphate-buffered saline, and sonicated. The His-tag Protein Purification Kit (Beyotime Biotechnology) was used, according to the manufacturer's instructions, to purify the recombinant MnArfn. The polyclonal antibody was prepared using the purified protein by Affinity Biosciences (Changzhou, China).

\subsection{Western blot analysis}

Proteins were isolated from the muscle, heart, hepatopancreas, gills, eyestalk, and stomach. Protein concentrations were determined with the Enhanced BCA Protein Assay Kit (Beyotime Biotechnology), according to the manufacturer's instructions. Samples (each containing $20 \mu \mathrm{g}$ of protein) were separated on $12 \%$ SDS-PAGE gels, and proteins were transferred onto nitrocellulose membranes. Membranes were blocked with QuickBlock $^{\mathrm{TM}}$ Blocking Buffer (Beyotime Biotechnology) and subsequently incubated with the polyclonal antibody against MnArfn. Membranes were washed 3 times with Tris-buffered saline containing $0.5 \%$ Tween-20 (TBST). Anti-rabbit horseradish peroxidase-conjugated IgG (1/1000) (Beyotime Biotechnology) was used as the secondary antibody. Membranes were washed 3 times with TBST. The MnArfn protein was visualized using the Affinity ${ }^{\circledR}$ ECL Kit (picogram) and quantified using a chemiluminescence imaging system (GDLDOC EQ, Bio-Rad).

\section{RESULTS}

\subsection{Sequence analysis}

The complete nucleotide sequence of MnArfn from Macrobrachium nipponense was determined by reverse-transcription PCR (RT-PCR) and RACE methods. The full-length cDNA of MnArfn was $1076 \mathrm{bp}$. It contained a $316 \mathrm{bp} 5^{\prime}$ untranslated region (UTR), a $537 \mathrm{bp} \mathrm{ORF,} \mathrm{and} \mathrm{a} 217$ bp 3' UTR with a poly(A) tail. The ORF encoded a 178 amino acid protein, while no signal peptide was found. The deduced molecular weight was $19.85 \mathrm{kDa}$, and the theoretical isoelectric point was 4.96. Furthermore, MnArfn had a conserved N-terminal myristoylation site of $\mathrm{G}_{2}$, P-loop $\left({ }^{24} \mathrm{GLDGVGKT}{ }^{31}\right)$, switch region $1\left({ }^{40} \mathrm{GKVVQTIPTIGF}{ }^{51}\right)$, interswitch region $\left({ }^{52} \mathrm{NVETVEYKNISFTVW}^{66}\right)$, and switch region 2 $\left({ }^{67}\right.$ DLPSQCKMRPLWRHY $\left.{ }^{81}\right)$. The cDNA sequence of the MnArfn gene was submitted to GenBank with accession number MN747148.1 (Fig. 1).

\subsection{Sequence alignment and phylogenetic analysis}

The deduced MnArfn amino acid sequence shared identities with Arfs from invertebrates and vertebrates, including Penaeus japonicus (60.67\%/Arfn), Litpenaeus vannamei (60.92\%/Arfn), Stylophora pistillata (59.78\%/Arf4), Sparus aurata (57.87\%/Arf4), Cimex lectularius (56.98\%/Arf2), Bigelowiella natans (55.62\%/Arfn), Rhinatrema bivittatum (60.34\%/ Arf5), and Anarrhichthys ocellatus (59.66\%/Arf4). Multiple sequence alignments of MnArfn with other known Arfs indicated that MnArfn was highly conserved and contained an N-terminal myristoylation site of $\mathrm{G}_{2}$. However, the protein sequences of the P-loop $\left({ }^{24}\right.$ GLDGVGKT $\left.{ }^{31}\right)$, switch region $1\left({ }^{40} \mathrm{GKVVQ}\right.$ TIPTIGF $\left.{ }^{51}\right)$, and switch region $2\left({ }^{67} \mathrm{DLPSQCKMR-}\right.$ PLWRHY ${ }^{81}$ ) of MnArfn differed from those of the other known Arf proteins (Fig. 2). In addition, Fig. 2 shows regions or sequences directly related to protein function, including the GXXXXGKT region, DVGG sequence, and NKQD sequence.

Phylogenetic analysis showed that MnAfrn clustered with Arf1 of $P$. japonicus, suggesting their evolutionary homology. MnAfrn did not cluster with the Japanese shrimp Afr4, indicating that MnAfrn may be a new Afr1 (Fig. 3). 
1 aacagatggcgtttatcgttcgcccgtgacagtttgtttacttccgacgttccccagaaa

61 cgcagtggattcttaacgatttgcgacaacaactaaagtgatatttcaccacagaaggac

121 aaaactaaagtgatatatcaccacagaagtgtagccgatggtcgagagtgataactagt

181 ggtagaagtggacggaggaacatatatagatacgagaacacactcaatctgatctcacgg

241 ctcggaactgtgaacgaaacagacgctatcgactgtttttgctgaacagattcaaatcat

301 tcaagttgcttcctagtgcataATGGGTGCCATCCTGAGCAGCATTCTGTCGCTATTTAA

$\begin{array}{llllllllllllllllllllll}14 & G & P & D & P & Y & R & I & V & M & V & G & L & D & G & V & G & K & T & T & I\end{array}$

361 GgGTCCTGATCCCTACAGGATAGTGATGGTCGGCCTCGATGGCGTCGGGAAGACGACGAT

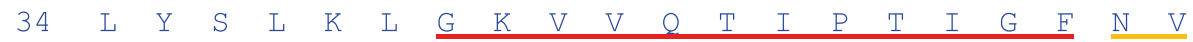

421 CCTGTACAGTCTCAAACTGGGCAAAGTTGTTCAGACCATCCCAACGATTGGGTTTAACGT

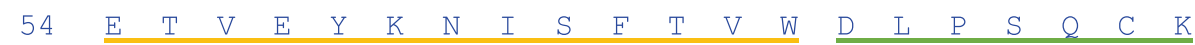

481 CGAGACGGTGGAGTACAAGAACATAAGTTTCACAGTGTGGGACCTTCCCTCCCAGTGCAA

$\begin{array}{lllllllllllllllllllll}74 & \mathrm{M} & \mathrm{R} & \mathrm{P} & \mathrm{L} & \mathrm{W} & \mathrm{R} & \mathrm{H} & \mathrm{Y} & \mathrm{F} & \mathrm{P} & \mathrm{G} & \mathrm{T} & \mathrm{T} & \mathrm{A} & \mathrm{A} & \mathrm{I} & \mathrm{F} & \mathrm{V} & \mathrm{V} & \mathrm{D}\end{array}$

541 GAtgCGTCCCTTGTGGAgGCATtATtTTCCAgGAACTACTGCCGCCATCTTCGTCGTAGA

$\begin{array}{llllllllllllllllllllllllllll}94 & S & T & D & K & E & R & L & P & E & S & R & E & A & L & Q & Y & V & L & D & E\end{array}$

601 CAgCACAGACAAGGAGAGATTACCGGAGTCAAGAGAAGCCTTACAATACGTGTTGGATGA

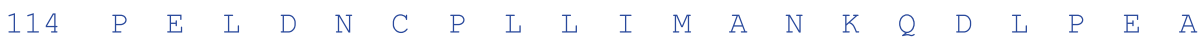

661 ACCAGAgCTGGATAACTGCCCTCTgCTCATAATGgCCAACAAGCAAGATCTACCAGAgGC $\begin{array}{lllllllllllllllllllllllllllllllll} & 134 & V & S & P & S & S & I & T & E & A & L & Q & L & E & R & L & K & R & P & W & F\end{array}$

721 TGTCTCGCCATCTTCCATCACAGAGGCCCTTCAACTCGAACGCCTGAAACGCCCTTGGTT $\begin{array}{llllllllllllllllllllllllllllll} & 54 & I & Q & G & T & S & A & L & E & S & T & G & I & C & E & A & L & D & W & L & A\end{array}$

781 CATCCAGGGCACCAGTGCCTTAGAATCGACGGGCATCTGCGAGGCTCTAGACTGGCTGGC

174 K A $\quad V \quad$ T $E$ *

841 CAAGGCGGTCACAGAGTGAgaaggattcggctgagttgtcaaaggattggtcttgattcg

901 tgtaagtttggggttatgcaaatagggtaatttaagtgcatgtttgaatgagggtacgtg

961 ttattataattccctcggggcctgagcctactcccaaggtaagggaattcagttgata

1021 attaggggtgtttttggcggagtagttttaattggaaaaaaaaaaaaaaaaaaa

Fig. 1. Nucleotide and amino acid sequences of MnArfn. Important sites are marked as follows; double red underline: codon (ATG) and termination codon (TAG); black arrowhead: N-terminal myristoylation site of $\mathrm{G}_{2}$; black underline: P-loop $\left({ }^{24}\right.$ GLDGVGKT $\left.{ }^{31}\right)$; red underline: switch region $1\left({ }^{40}\right.$ GKVVQTIPTIGF $\left.^{51}\right)$; yellow underline: interswitch region $\left({ }^{52} \mathrm{NVETVEYKN-}\right.$ SFTVW $\left.^{66}\right)$; green underline: switch region $2\left({ }^{67}\right.$ DLPSQCKMRPLWRHY $\left.{ }^{81}\right)$

\subsection{Tissue distribution of MnArfn}

qPCR and Western blotting were performed to examine the tissue distribution and expression of MnArfn. The results of qPCR showed that MnArfn was expressed in all examined tissues, including the muscle, heart, gills, hepatopancreas, eyestalk, and stomach. The highest expression was detected in the hepatopancreas, followed by the gills, whereas the lowest expression was detected in the eyestalk (Fig. 4A). Western blotting results also showed that MnArfn was expressed in all examined tissues. Here as well, the highest level was observed in the hepatopancreas, whereas the lowest level was in the eyestalk (Fig. 4B).

\section{4. mRNA expression of MnArfn in hepatopan- creas and gills after ammonia nitrogen stress}

The temporal expression of MnArfn in hepatopancreas and gills of $M$. nipponense under ammonia nitrogen stress was examined by qPCR. Compared with the control group, the MnArfn level in the 


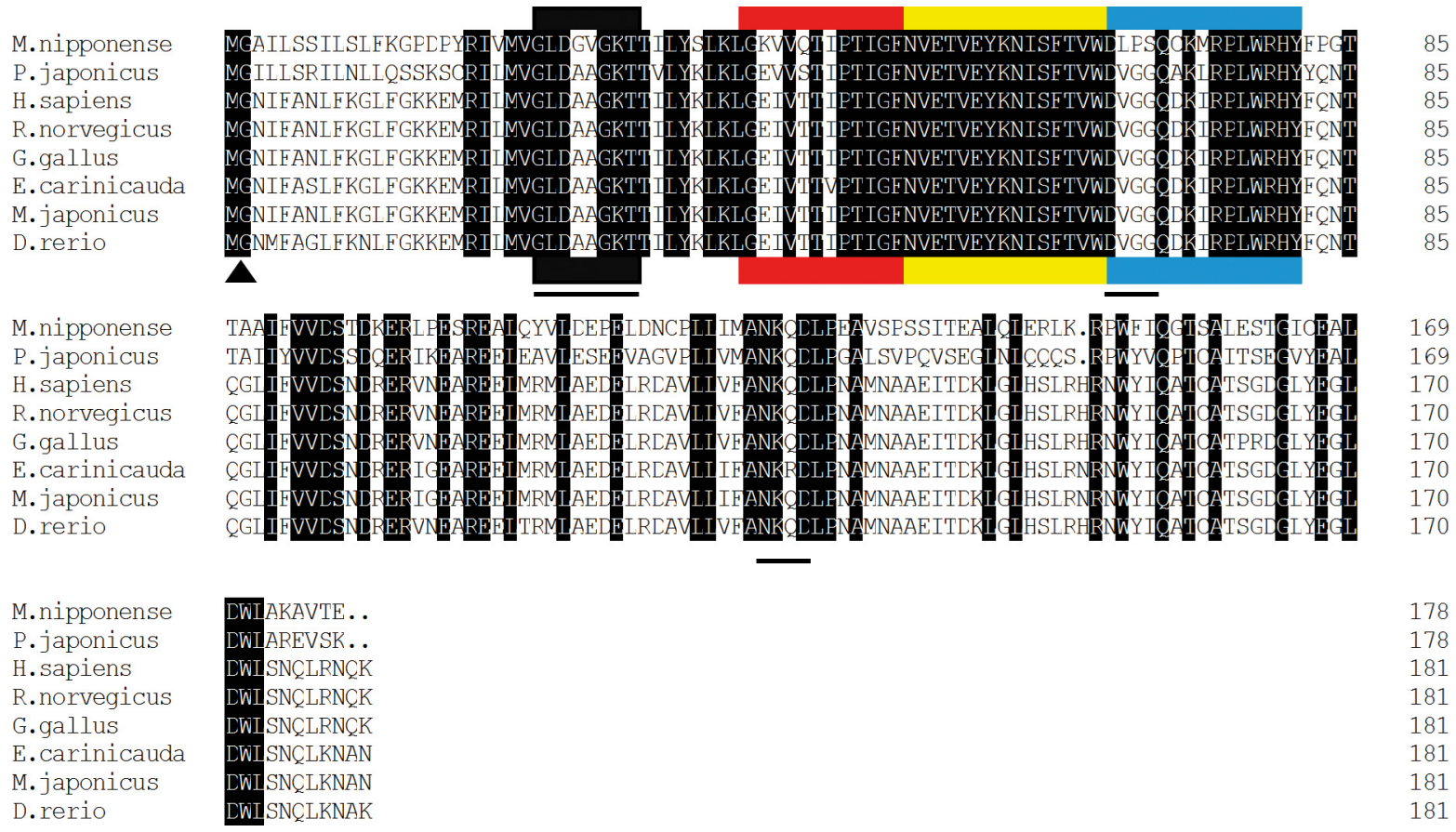

Fig. 2. Partial results of multiple alignment of MnArfn with other proteins of the Arf family: Penaeus japonicus (GenBank accession number ADE42874.1), Homo sapiens (NP_001649.1), Rattus norvegicus (NP_071963.1), Gallus gallus (NP_00100 6352.1), Exopalaemon carinicauda (AQM40226.1), Marsupenaeus japonicus (ADE42873.1), Danio rerio (AAH66632.1). The P-loop (24GLDGVGKT31), switch region 1 (40GKVVQTIPTIGF51), interswitch region (52NVETVEYKNISFTVW66), and switch region 2 (67DLPSQCKMRPLWRHY81) are shown as a black, red, yellow, and blue regions, respectively. GXXXXGKT region, DVGG sequence, and NKQD sequence are marked with black underlines

hepatopancreas increased in response to ammonia nitrogen stress, with the highest level measured at $6 \mathrm{~h}(\mathrm{p}<0.01)$, indicating that ammonia nitrogen stress can induce MnArfn expression. The MnArfn level decreased at $12 \mathrm{~h}$ and reached the lowest level at $72 \mathrm{~h}$ (Fig. 5A). The MnArfn level was significantly increased in the gills at $6 \mathrm{~h}(\mathrm{p}<0.01)$. Subsequently, it decreased at $12 \mathrm{~h}$ and reached the lowest level at 72 h (Fig. 5B).

\subsection{Recombinant expression and purification}

Recombinant MnArfn expression was induced by IPTG, and it was overexpressed as a soluble protein in E. coli BL21 (DE3) cells at $25^{\circ} \mathrm{C}$. The deduced molecular weight of recombinant MnArfn was $19.85 \mathrm{kDa}$, consistent with the SDS-PAGE results (Fig. 6).

\section{DISCUSSION}

With the rapid development of intensive aquaculture models, the content of ammonia nitrogen in aquaculture environments has become the second major factor restricting the development of aquaculture besides dissolved oxygen (Ebeling et al. 2006). The production of ammonia nitrogen comes not only from the process of protein metabolism in aquatic animals, but also from the decomposition of feed by microorganisms in the culture environment (Chang et al. 2015). Previous studies have demonstrated that suboptimal environmental conditions can weaken the immune function of crustaceans and increase their susceptibility to certain diseases and infections (Li \& Chen 2008). Ammonia nitrogen can induce oxidative stress by increasing the generation of reactive oxygen species (Chang et al. 2015). Furthermore, ammonia nitrogen stress can decrease peroxinectin and prophenoloxidase gene levels by 50 and $60 \%$, respectively, in Penaeus stylirostris (Le Moullac \& Haffner 2000). High concentrations of ammonia nitrogen can suppress immune function in shrimp, thereby damaging their organs (Cheng \& Chen 2002). We previously reported that high concentrations of ammonia nitrogen impair organs and the immune system of prawns (Sun et al. 2020). To sum up, a high concentration of ammonia nitrogen in the aquaculture environment directly impairs the health 


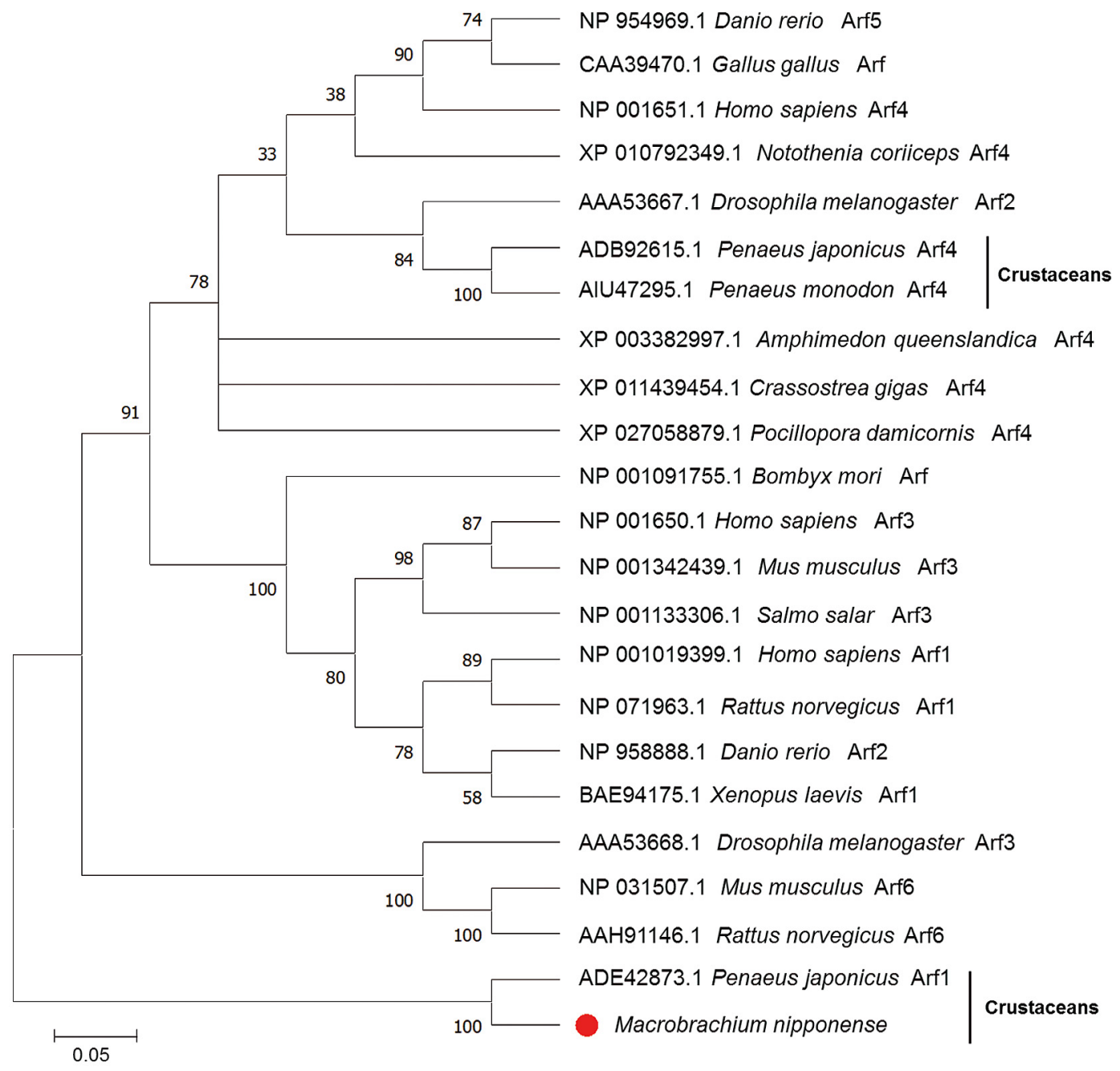

Fig. 3. Phylogenetic analysis of the Arf family from different species, along with their GenBank accession numbers

of aquatic animals, especially Macrobrachium nipponense, and further affects the healthy development of the production and aquaculture industry.

The hepatopancreas of prawns is comparable to the fat of insects and the liver of mammals, which serves as a sensitive indicator of the environment and the general health of organisms (Sousa \& Petriella 2000, Gross et al. 2001). Gills, as the respiratory organ of prawns, are in direct contact with freshwater; thus, they provide the first-line defense and play a crucial role in the response to biotic and abiotic factors (Cui et al. 2017). Therefore, genes that are either up- or down-regulated in response to ammonia nitrogen stress should be studied to obtain a better understanding of how hepatopancreas and gills respond to environmental toxicants.

Studying the interacting mechanisms between aquatic organisms and environments, such as aquatic environment changes and bacterial and viral infection, is very important for aquatic production. Arfs reported in Penaeus monodon may be closely related to ammonia nitrogen stress and the immune process of pathogenic infection (Duan et al. 2016). Similar reports suggested that Arfs may be associated with viral infections (Zhang et al. 2010). Arfs are members of the Ras gene superfamily and are highly conserved in biological evolution, especially the N-terminal myristoylation site of G2 and the interswitch region. The P-loop is also expressed as the GXXXXGKT region (human Arf1 [hArf1], 24th-31st amino acids) associated with GTP dissociation. In this study, although the amino acid sequence of MnArfn is not identical to that of hArf1, the amino acid at the key position has not changed, and the third amino acid in this region is not glycine which plays a key role in the dissociation of GTP in normal Ras genes; 


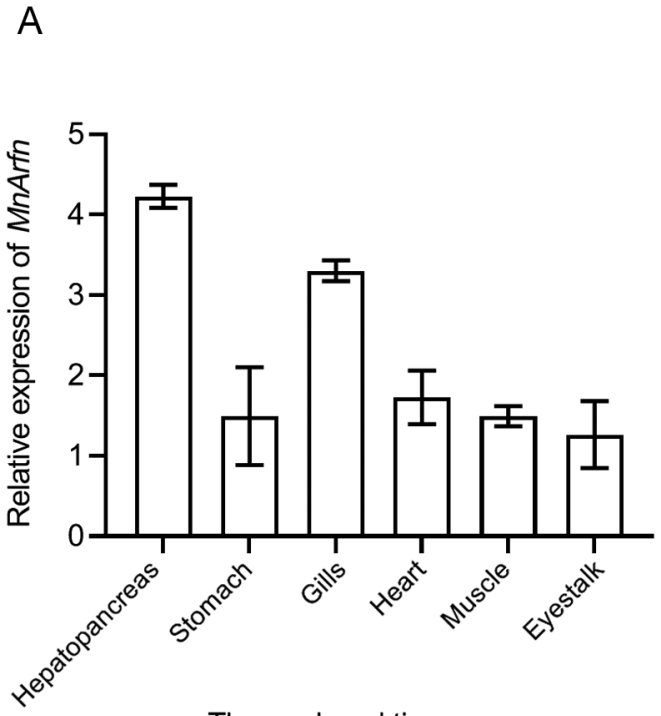

The analyzed tissues

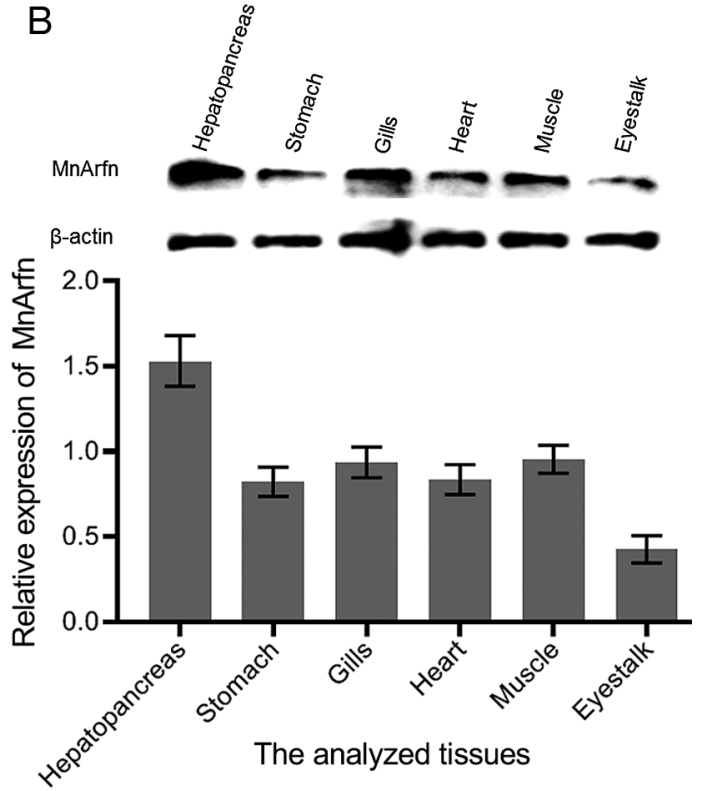

Fig. 4. Expression of MnArfn in different tissues of Macrobrachium nipponense and analyzed by (A) qPCR and (B) Western blotting. $\beta$-actin was used as the control; data are shown as means $\pm \mathrm{SE}(\mathrm{N}=3)$
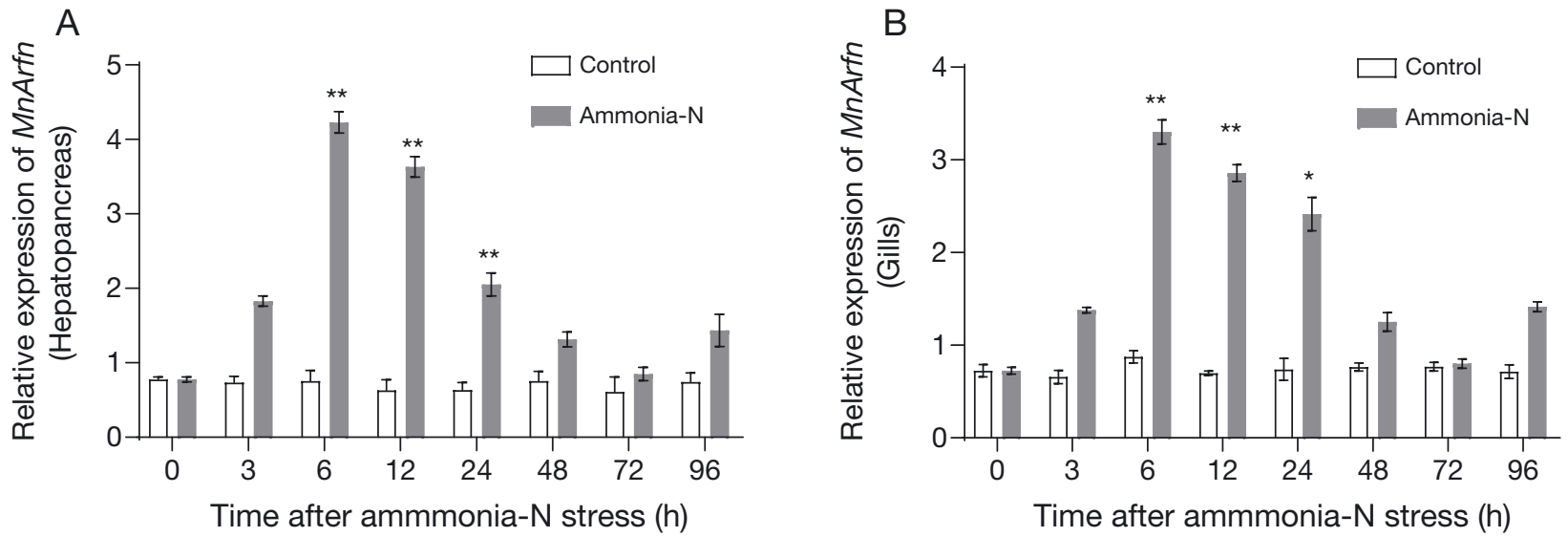

Fig. 5. Expression of MnArfn in (A) hepatopancreas and (B) gills of Macrobrachium nipponense at different times after ammonia nitrogen stress. Data are shown as means $\pm \mathrm{SE}(\mathrm{N}=6)$. Asterisks represent significant differences $\left({ }^{*} \mathrm{p}<0.05,{ }^{* *} \mathrm{p}<0.01\right)$

therefore, we speculated that it does not affect the biological activity of this region (Botstein et al. 1988). The DVGG sequence (hArf1, 67-70th amino acids) in switch region 2 is thought to be involved in the regulation of GTP binding to $\mathrm{Mg}^{2+}$, but the MnArfn sequence is not completely identical, suggesting that it may cause differential binding activities between GTP and $\mathrm{Mg}^{2+}$. The NKQD sequence (hArf1, 126129th amino acid) is thought to be involved in binding with the guanine ring, and MnArfn is consistent with all species to which it was compared.

Most of the research on the structure and function of Arfs has been conducted by investigating its mutants, which also provides a novel approach to study the functional changes caused by the sequence changes in switch region 2 that differ from other Arfs. In this study, the full-length cDNA of MnArfn was identified from $M$. nipponense, and this is the first report on the involvement of the MnArfn gene in the ammonia nitrogen stress response of $M$. nipponense. Sequence and phylogenetic analyses indicated that MnArfn belongs to the Arf family and showed 55-62\% identity with Arfs of other species. The phylogenetic tree, which included Arfs of crustaceans, mammals, fish, birds, and other animals, showed that MnArfn had high homology with the Arf1 of Penaeus 


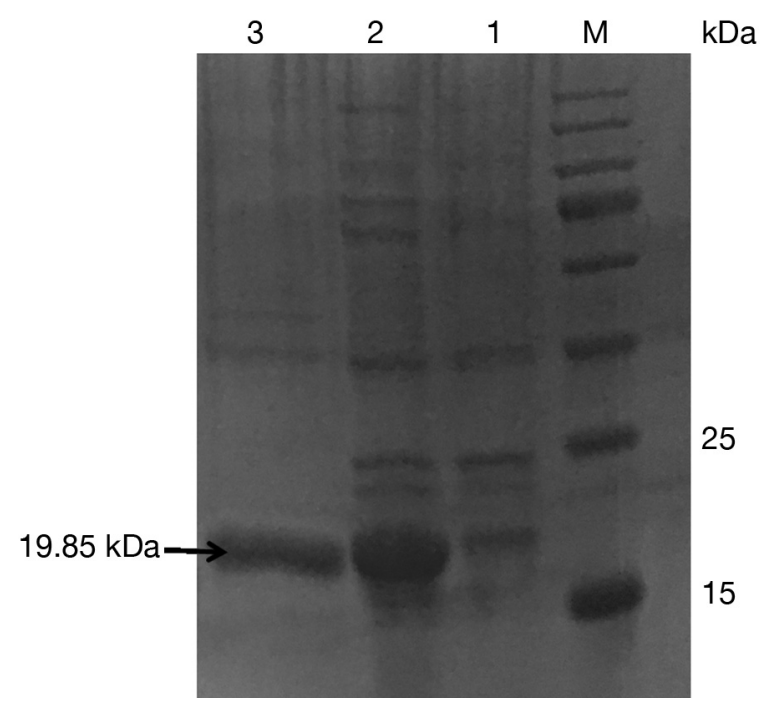

Fig. 6. SDS-PAGE analysis of recombinant MnArfn. Lane M: Protein marker; lane 1: expression without IPTG induction; lane 2: induced MnArfn-pET30a; lane 3: purified recombinant MnArfn protein

japonicas (Fig. 3). These findings suggest that MnArfn is a new member of the Arf family in M. nipponense. We also hypothesize that MnArfn plays an important role in the ammonia stress response.

To examine the function of MnArfn in M. nipponense, its expression in tissues was examined by qPCR. MnArfn was expressed in all examined tissues, especially in hepatopancreas and gills, consistent with the tissue distribution of Arfs in Macrobrachium rosenbergii (Arf1/2) (Ding et al. 2015), Exopalaemon carinicauda (Arf1) (Duan et al. 2016), and Penaeus monodon (Arf4) (Shekhar \& Gomathi 2017), indicating that MnArfn has many functions. In this study, we observed a significant increase of MnArfn expression in hepatopancreas and gills under conditions of ammonia nitrogen stress. As important immune organs, hepatopancreas and gills function in the first-line defense against environmental stressors and pathogens (Vallet-Gely et al. 2008, Huang \& Ren 2015). Previous studies have reported that immune-related genes, such as a2M (Ho et al. 2009), NM23 (Duan et al. 2015), HSP90 (Zhao et al. 2011), and CTL (Xiu et al. 2015), are highly expressed in hepatopancreas and gills of prawns and shrimps. The high expression of MnArfn in hepatopancreas and gills may indicate that it plays an important role in the ammonia stress response. Under conditions of ammonia nitrogen stress, the MnArfn level was significantly up-regulated, reaching its highest and lowest levels at 6 and $72 \mathrm{~h}$, respectively. This immediate increase in the MnArfn level after exposure to ammonia nitrogen stress indicates that the protein functions in the early response by suppressing stress. Western blotting results also indicated that MnArfn was expressed in all examined tissues, again with the highest levels in the hepatopancreas, followed by the gills, consistent with the qPCR results. Taken collectively, MnArfn may protect prawns from ammonia nitrogen stress and plays an essential role in regulating ammonia stress of $M$. nipponense.

\section{CONCLUSION}

This is the first report of the identification and cloning of the Macrobrachium nipponense Arfn gene MnArfn. The results of this study provide a foundation for further investigations of the stress tolerance mechanism of the MnArfn gene. MnArfn was highly expressed in hepatopancreas and gills. Under ammonia nitrogen stress, the expression of MnArfn in metabolism-related tissues showed that the expression of MnArfn could be induced and might play a role in the stress regulation of prawns. These results provide a new avenue and theoretical basis for follow-up studies on the mechanism(s) of crustacean resistance to external adverse environments and provide a new perspective to screen for stress-resistant individuals and cultivating more resistent varieties.

Acknowledgements. This work was financially supported by the Shandong Provincial Agricultural Good Breeding Project (Selection and Breeding of Breakthrough New Varieties of High Quality and Highly Resistant Shrimp, No. 2019 LZGC014); Shandong Province 'Bohai Sea Granary' Science and Technology Demonstration Project (Integrated Demonstration of Ecological Symbiosis Agricultural Model Innovation and Synergistic Technology in Saline Wetland, No. 2019BHLC006); Shandong Provincial Agricultural Good Seed Project (No. 2017LZN010); Shandong Provincial Agricultural Science and Technology Fund (Industrial Upgrading Project in the Park) Project (Construction of Dongping [Yellow River Bank District] Fish and Shrimp Breeding Innovation Base and Research and Demonstration on Poverty Alleviation in Science and Technology Industries, No. 2019 YQ032); Shandong Province Modern Agricultural Industrial Technology System Shrimp and Crab Innovation Team (SDAIT-13-02); and Shandong Postgraduate Education Innovation Project (SDYY11083; SDYC12048).

\section{LITERATURE CITED}

Botstein D, Segev N, Stearns T, Hoyt MA, Holden J, Kahn RA (1988) Diverse biological functions of small GTPbinding proteins in yeast. Cold Spring Harb Symp Quant Biol 53:629-636 
Chang ZW, Chiang PC, Cheng W, Chang CC (2015) Impact of ammonia exposure on coagulation in white shrimp, Litopenaeus vannamei. Ecotoxicol Environ Saf 118: 98-102

Chen CY, Chen PC, Weng FCH, Shaw GTW, Wang D (2017) Habitat and indigenous gut microbes contribute to the plasticity of gut microbiome in oriental river prawn during rapid environmental change. PLOS ONE 12:e0181427

* Chen JC, Kou YZ (1992) Effects of ammonia on growth and molting of Penaeus japonicus juveniles. Aquaculture 104:249-260

* Chen JC, Lin CY (1992) Oxygen consumption and ammonia nitrogen excretion of Penaeus chinensis juveniles exposed to ambient ammonia at different salinity levels. Comp Biochem Physiol C Comp Pharmacol 102:287-291

Chen JS, Zhou F, Huang JH, Ma ZH, Jiang SG, Qiu LH, Qin JG (2016) Ammonia and salinity tolerance of Penaeus monodon across eight breeding families. Springerplus 5: 171

Cheng W, Chen JC (2002) The virulence of Enterococcus to freshwater prawn Macrobrachium rosenbergii and its immune resistance under ammonia stress. Fish Shellfish Immunol 12:97-109

Cui F, Yu Y, Bao F, Wang S, Xiao MS (2018) Genetic diversity analysis of the oriental river prawn (Macrobrachium nipponense) in Huaihe River. Mitochondrial DNA A DNA Mapp Seq Anal 29:737-744

Cui Y, Ren X, Li J, Zhai Q, Feng Y, Xu Y, Ma L (2017) Effects of ammonia-N stress on metabolic and immune function via the neuroendocrine system in Litopenaeus vannamei. Fish Shellfish Immunol 64:270-275

* D'Souza-Schorey C, Chavrier P (2006) ARF proteins: roles in membrane traffic and beyond. Nat Rev Mol Cell Biol 7: 347-358

* Ding ZF, Ren J, Tan JM, Wang Z, Yin SW, Huang Y (2015) Characterization of two novel ADP ribosylation factors from giant freshwater prawn Macrobrachium rosenbergii and their responses to WSSV challenge. Dev Comp Immunol 48:204-209

Donaldson JG, Honda A (2005) Localization and function of Arf family GTPases. Biochem Soc Trans 33:639-642

* Duan Y, Liu P, Li J, Li J, Chen P (2013) Expression profiles of selenium dependent glutathione peroxidase and glutathione S-transferase from Exopalaemon carinicauda in response to Vibrio anguillarum and WSSV challenge. Fish Shellfish Immunol 35:661-670

* Duan Y, Li J, Zhang Z, Li J, Liu P (2015) The role of oncoprotein NM23 gene from Exopalaemon carinicauda is [sic] response to pathogens challenge and ammonia nitrogen stress. Fish Shellfish Immunol 47:1067-1074

Duan Y, Li J, Zhang Z, Li J, Liu P (2016) Characterization of ADP ribosylation factor 1 gene from Exopalaemon carinicauda and its immune response to pathogens challenge and ammonia nitrogen stress. Fish Shellfish Immunol 55: $123-130$

* Ebeling JM, Timmons MB, Bisogni JJ (2006) Engineering analysis of the stoichiometry of photoautotrophic, autotrophic, and heterotrophic removal of ammonia-nitrogen in aquaculture systems. Aquaculture 257:346-358

* Gross PS, Bartlett TC, Browdy CL, Chapman RW, Warr GW (2001) Immune gene discovery by expressed sequence tag analysis of hemocytes and hepatopancreas in the pacific white shrimp, Litopenaeus vannamei, and the Atlantic white shrimp, L. setiferus. Dev Comp Immunol 25:565-577
He N, Liu H, Xu X (2004) Identification of genes involved in the response of haemocytes of Penaeus japonicus by suppression subtractive hybridization (SSH) following microbial challenge. Fish Shellfish Immunol 17:121-128

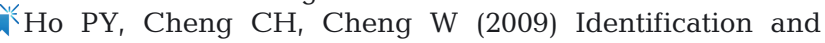
cloning of the $\alpha 2$-macroglobulin of giant freshwater prawn Macrobrachium rosenbergii and its expression in relation with the molt stage and bacteria injection. Fish Shellfish Immunol 26:459-466

*Huang Y, Ren Q (2015) Identification and function of 11 Rab GTPases in giant freshwater prawn Macrobrachium rosenbergii. Fish Shellfish Immunol 43:120-130

* Le Moullac G, Haffner P (2000) Environmental factors affecting immune responses in Crustacea. Aquaculture 191:121-131

Li CC, Chen JC (2008) The immune response of white shrimp Litopenaeus vannamei and its susceptibility to Vibrio alginolyticus under low and high $\mathrm{pH}$ stress. Fish Shellfish Immunol 25:701-709

Li J, Han J, Chen P, Chang Z and others (2012) Cloning of a heat shock protein 90 (HSP90) gene and expression analysis in the ridgetail white prawn Exopalaemon carinicauda. Fish Shellfish Immunol 32:1191-1197

Kiang Z, Liu R, Zhao D, Wang L, Sun M, Wang M, Song L (2016) Ammonia exposure induces oxidative stress, endoplasmic reticulum stress and apoptosis in hepatopancreas of Pacific white shrimp (Litopenaeus vannamei). Fish Shellfish Immunol 54:523-528

iu W, Han F, Zhang X (2009) Ran GTPase regulates hemocytic phagocytosis of shrimp by interaction with myosin. J Proteome Res 8:1198-1206

* Lu X, Kong J, Luan S, Dai P, Meng X, Cao B, Luo K (2016) Transcriptome analysis of the hepatopancreas in the Pacific white shrimp (Litopenaeus vannamei) under acute ammonia stress. PLOS ONE 11:e0164396

Moraes-Valenti PMC, Valenti WC (2007) Effect of intensification on grow out of the Amazon river prawn, Macrobrachium amazonicum. J World Aquacult Soc 38: 516-526

* Myers KR, Casanova JE (2008) Regulation of actin cytoskeleton dynamics by Arf-family GTPases. Trends Cell Biol 18:184-192

*Pan D, He N, Yang Z, Liu H, Xu X (2005) Differential gene expression profile in hepatopancreas of WSSV-resistant shrimp (Penaeus japonicus) by suppression subtractive hybridization. Dev Comp Immunol 29:103-112

* Price C, Black KD, Hargrave BT, Morris JA Jr (2015) Marine cage culture and the environment: effects on water quality and primary production. Aquacult Environ Interact 6: 151-174

* Shekhar MS, Gomathi A (2017) Molecular characterization of differentially expressed ADP ribosylation factor from WSSV infected black tiger shrimp Penaeus monodon. Proc Natl Acad Sci India Sect B (Biol Sci) 87:827-837

* Sousa LG, Petriella AM (2000) Histology of the hepatopancreas of the freshwater prawn Palaemonetes argentinus (Crustacea, Caridea). Biocell 24:189-195

* Sun B, Luo H, Zhao S, Yu JL, Lv XT, Yi C, Wang H (2019) Characterization and expression analysis of a $\mathrm{gC} 1 \mathrm{qR}$ gene from Macrobrachium nipponense under ammonia nitrogen stress. Aquaculture 513:734426

* Sun B, Luo H, Zhao S, Lv XT, Yi C, Chen D, Zeng Q (2020) Characterization and the role of $\alpha 2$-macroglobulin from Macrobrachium nipponense in response to ammonia nitrogen stress. Aquaculture 522:735128 
Vallet-Gely I, Lemaitre B, Boccard F (2008) Bacterial strategies to overcome insect defences. Nat Rev Microbiol 6: 302-313

Wang H, Ma J, Ruan L, Xu X (2009) Cloning of a centaurin$\alpha 1$ like gene MjCent involved in WSSV infection from shrimp Marsupeneaus japonicus. Fish Shellfish Immunol 26:279-284

Wu W, Zong R, Xu J, Zhang X (2008) Antiviral phagocytosis is regulated by a novel Rab-dependent complex in shrimp Penaeus japonicus. J Proteome Res 7: $424-431$

Xiu Y, Hou L, Liu X, Wang Y, Gu W, Meng Q, Wang W (2015) Isolation and characterization of two novel C-type lectins from the oriental river prawn, Macrobrachium nipponense. Fish Shellfish Immunol 46:603-611

Yu J, Sun J, Zhao S, Wang H, Zeng Q (2019) Transcriptome

Editorial responsibility: Vengatesen Thiyagarajan, Hong Kong SAR

Reviewed by: 3 anonymous referees analysis of oriental river prawn (Macrobrachium nipponense) hepatopancreas in response to ammonia exposure. Fish Shellfish Immunol 93:223-231

Y Yue F, Pan L, Xie P, Zheng D, Li J (2010) Immune responses and expression of immune-related genes in swimming crab Portunus trituberculatus exposed to elevated ambient ammonia-N stress. Comp Biochem Physiol A Mol Integr Physiol 157:246-251

* Zhang M, Ma J, Lei K, Xu X (2010) Molecular cloning and characterization of a class II ADP ribosylation factor from the shrimp Marsupenaeus japonicus. Fish Shellfish Immunol 28:128-133

Z Zhao W, Chen L, Qin J, Wu P, Zhang F, Li E, Tang B (2011) MnHSP90 cDNA characterization and its expression during the ovary development in oriental river prawn, Macrobrachium nipponense. Mol Biol Rep 38:1399-1406

Submitted: September 20, 2020

Accepted: February 17, 2021

Proofs received from author(s): May 2, 2021 